\title{
Klebsiella pneumoniae NdpA suppresses ERK pathway-mediated host early inflammatory responses and is degraded through the ubiquitin-proteasome pathway
}

Dear Editor,

Klebsiella pneumoniae (KP) is an important opportunistic pathogen causing community-acquired and nosocomial infections. When the host is immunocompromised, the pathogen would infect the host and cause diseases, such as pneumoniae, sepsis, liver abscess, meningitis, urinary tract inflammation and wound infection (Karaiskos et al., 2016; Park et al., 2015). The phenomenon that $K$. pneumoniae has a preference to infect immunocompromised populations, especially seniors suggests that the outcomes of $K$. pneumoniae infection depend on the pathogen-host interactions, but up to now, the molecular mechanisms underlying $K$. pneumoniae-host interactions remain largely unknown.

Previous studies reported that $K$. pneumoniae suppresses inflammatory cytokine production during early period of infection (Lawlor et al., 2006), and this bacteria can block the activation of inflammatory responses by antagonizing NF-KB and MAPK signaling pathways (Frank et al., 2013; Regueiro et al., 2011). We discovered that the $K$. pneumoniae nucleoid-associated protein (NdpA) is highly conserved among gram-negative bacteria. Transient expression of NdpA in human embryonic kidney HEK239T cells inhibited the Elk activation induced by RasV12, as well as V-Raf (constitutive active Raf) and MEK1-ED (constitutive active MEK1), respectively (Fig. 1A-C). K. pneumoniae NdpA also promoted tumor necrosis factor (TNF) $\alpha$-stimulated NF-kB activation (Fig. S1A), and had little, if any, inhibitory effect on JNK and p38 signaling pathways (Fig. S1B). Given the lack of inflammation at the early stage of $K$. pneumoniae infection (Lawlor et al., 2006), we thus focused on the elucidation of the suppressive effects of $K$. pneumoniae NdpA on ERK signaling pathway-mediated host early inflammatory responses. Because many pathogenic bacteria have secretion systems to inject their virulence factors into host cells to interfere their functions. Thus, we sought to examine whether K. pneumoniae NdpA could be secreted into host cells during infection. Immunoblot analysis showed that NdpA entered into the cytosol of the human alveolar epithelial cells A549 during $K$. pneumoniae infection (Fig. S2A). In addition, we found that the phosphorylation of ERK $1 / 2$ activated by MEK1-ED was largely reduced by NdpA (Fig. 1D). Consistently, NdpA also abolished extracellular stimuli epidermal growth factor (EGF)-activated ERK1/2 phosphorylation (Fig. 1E). To further confirm the role of NdpA in the suppression of ERK signaling during K. pneumoniae infection, we tried to knockout the gene encoding NdpA with several methods available, but after many attempts we failed to obtain the expected mutant strain. We thus adopted the alternative strategy to investigate the host immune-regulatory function of NdpA by overexpressing it in K. pneumoniae. We found that overexpression of NdpA in K. pneumoniae and $E$. coli resulted in down-regulation of phospho-ERK $1 / 2$ (p-ERK1/2) in A549 cells during $K$. pneumoniae infection (Figs. 1F and S2B).

With a central role in recruiting and infiltrating neutrophils into inflammatory sites, IL8 is known as a main inflammatory molecule involved in host defense against $K$. pneumoniae infection (Harada et al., 1994). We thus next explored whether NdpA regulates the expression of IL8 in human alveolar epithelial cells during $K$. pneumoniae infection. The data from quantitative real-time PCR showed that compared with the wild-type (WT) $K$. pneumoniae strain, the NdpA-overexpressing $K$. pneumoniae strain significantly down-regulated the mRNA of IL8 in A549 cells (Fig. 1G). Accordingly, the secretion of IL8 was apparently attenuated by the overexpression of NdpA in K. pneumoniae as analyzed by enzyme-linked immunosorbent assay (ELISA) (Fig. $1 \mathrm{H}$ ). To determine whether the inhibitory effects of NdpA on IL8 production is dependent on ERK signaling pathway, we pretreated A549 cells with U0126, a specific inhibitor of ERK pathway, before the infection assay, and we found that the WT K. pneumoniae strain and the NdpA-overexpressing $K$. pneumoniae strain showed similar amount of IL8 production during infection of A549 cells (Fig. S3A and S3B). 

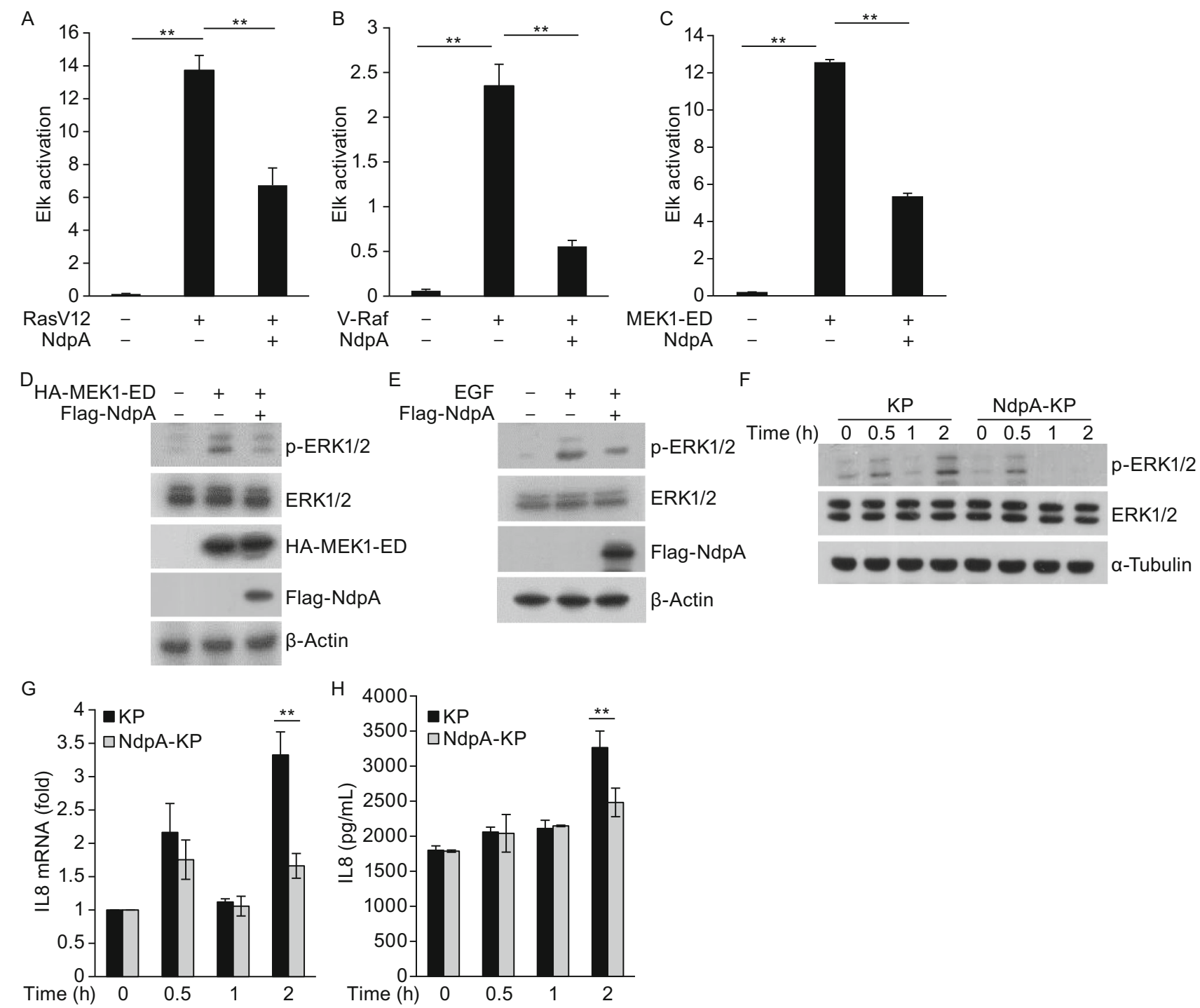

Figure 1. K. pneumoniae NdpA inhibits ERK1/2 signaling pathway activation. (A-C) Dual-luciferase assays of RasV12 (A)-, V-Raf (B)-, and MEK1-ED (C)- activated ERK1/2 signaling in the absence or presence of NdpA. Data are representative of at least three independent experiments (mean and SEM). ${ }^{*} P<0.05$, ${ }^{*} P<0.01$ (two-tailed unpaired $t$-test). (D-F) The effects of NdpA on ERK1/2 phosphorylation as assessed by immunoblotting. HEK293T cells expressing MEK1-ED without or with NdpA (D); HEK293T cells transfected with empty vector or vector encoding flag-tagged NdpA were treated with $100 \mathrm{ng} / \mathrm{mL}$ of EGF for $15 \mathrm{~min}$ (E); A549 cells were infected with WT or NdpA-overexpressing K. pneumoniae (NdpA-KP) for indicated time (F). (G) Quantitative real-time PCR analysis of $/ 18$ mRNA in A549 cells infected with KP or NdpA-KP for indicated time. (H) ELISA analysis of IL8 in the supernatants of A549 cells infected with KP or NdpA-KP for indicated time, respectively.

We further sought to investigate the underlying mechanisms by which NdpA suppresses host inflammatory responses. Based on the observation that NdpA inhibited ERK1/2 phosphorylation stimulated by MEK1-ED, the activator of ERK pathway next to ERKs (Fig. 1D), MEK1 and ERKs were chosen to verify their interactions with NdpA. HEK293T cells expressing Flag-tagged ERK2 or Myc-tagged MEK1 were used for co-immunoprecipitation analysis with
His-tagged NdpA purified from E. coli BL21, and the data indicated that NdpA could specifically bind to ERK2, but not MEK1 (Fig. S4A). Furthermore, we found that overexpression of NdpA in HEK293T cells completely abolished the binding of MEK1 to ERK2 (Fig. S4B).

Through protein sequence alignment analysis, we found that NdpA harbors a canonical D motif (15-KRDEQNLEL-23) for ERKs docking. The $D$ motif contains the consensus 


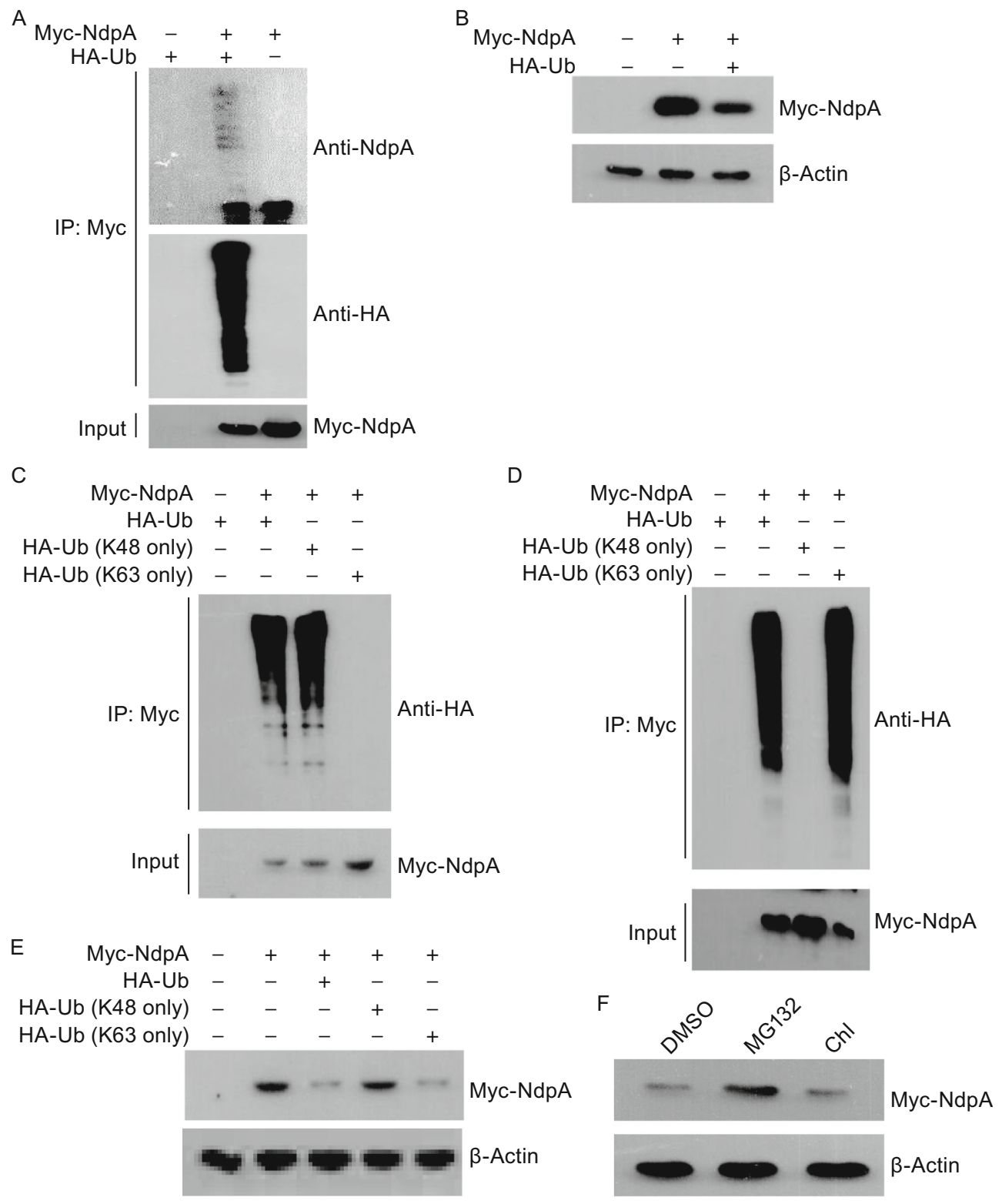

Figure 2. K. pneumoniae NdpA is degraded through the ubiquitin-proteasome pathway in host cells. (A) Ubiquitination assay of NdpA in vivo. HEK293T cells were transfected with indicated plasmids, $24 \mathrm{~h}$ later, Myc-NdpA was immunuoprecipated with the gelconjugated Myc antibody and immunoblotted with anti-HA and anti-NdpA antibodies. (B) Immunoblotting analysis of Myc-NdpA in the absence or presence of HA-Ub in HEK293T cells. (C) HEK293T cells expressing Myc-NdpA together with HA-Ub or Lys 48-linked polyubiquitylation (K48 only) or Lys 63-linked polyubiquitylation (K63 only) were subjected to the gel-conjugated Myc antibody and immunoblotted with HA antibody. (D) HEK293T cells expressing Myc-NdpA together with HA-Ub or HA-Ub (K48R) or HA-Ub (K63R) were subjected to the gel-conjugated Myc antibody and immublotted with HA antibody. (E) A549 cells transfected with indicated plasmids were detected for NdpA protein by immunoblotting. (F) A549 cells expressing Myc-NdpA were treated with DMSO or 10 $\mu \mathrm{mol} / \mathrm{L}$ MG132 or $100 \mu \mathrm{mol} / \mathrm{L}$ chroloquine $(\mathrm{Chl})$ for $6 \mathrm{~h}$, and then NdpA protein was detected by immunoblotting.

sequence $(\mathrm{K} / \mathrm{R})_{1-2^{-}}(\mathrm{X})_{2-6}-\varnothing_{\mathrm{A}}-\mathrm{X}-\varnothing_{\mathrm{B}} \quad\left(\varnothing_{\mathrm{A}}\right.$ and $\varnothing_{\mathrm{B}}$ are hydrophobic residues) (Supplementary Fig. 4C) (Zhou et al., 2006; Zhu et al., 2007). To identify which site plays the most critical role in the binding to ERK1/2, four mutants of $\mathrm{NdpA}$ were constructed, including $\mathrm{NdpA}(\mathrm{K} 15 \mathrm{~A})$, NdpA (R16A), NdpA (L21E) and NdpA (L23E). Through dual-luciferase assay, we found that $\mathrm{NdpA}$ (L21E) evidently impaired the inhibitory effects of NdpA on ERK pathway (Fig. S4D). His- 
tag pull-down analysis further confirmed that NdpA could directly bind to ERK2 in vitro, but the NdpA (L21E) mutant had little binding affinity (Supplementary Fig. 4E). Consistently, $\operatorname{NdpA}(\mathrm{L} 21 \mathrm{E})$ lost the ability to block the interactions between ERK2 with MEK1 (Fig. S4F). Unlike WT NdpA, NdpA (L21E) could not efficiently inhibit MEK1-ED-stimulated ERK1/2 phosphorylation in HEK293T (Supplementary Fig. 5A), and also failed to inhibit ERK1/2 phosphorylation in A549 cells during K. pneumoniae infection (Supplementary Fig. 5B). Consistently, quantitative real-time PCR and ELISA analysis also showed that $\mathrm{NdpA}$ (L21E) couldn't effectively downregulate IL8 expression in A549 cells during $K$. pneumoniae infection (Fig. S5C and S5D). Together, these data indicate that NdpA inhibited ERK signaling pathway-mediated inflammation in a $D$ motif-dependent manner.

Since the suppressive effects of $K$. pneumoniae are usually attenuated at about 4 hours post infection of host cells, we thus further questioned whether the host could counteract the regulatory effects of NdpA during the course of $K$. pneumoniae infection by regulating the protein stability of NdpA. Interesting, by online bioinformatics analysis (http:// smart.embl-heidelberg.de/), we identified two tandem ubiquitin (Ub)-interacting Motifs (UIMs) in K. pneumoniae NdpA. The UIM motif has been reported to occur more frequently in eukaryotic cells instead of prokaryotic cells and it is specific for interacting with free Ub, poly-ubiquitin chains, as well as ubiquitin-conjugated proteins (Polo et al., 2003). Therefore, we hypothesized that NdpA might be degraded by the host through an ubiquitin-dependent pathway. When the vectors encoding $\mathrm{NdpA}$ and ubiquitin were co-transfected in HEK293T cells for co-immunoprecipitation analysis, we observed that NdpA was polyubiquitinated in vivo (Fig. 2A). Furthermore, overexpression of ubiquitin in HEK293T cells caused the reduction of NdpA protein (Fig. 2B). To identify whether the two UIMs play a role in the polyubiquitination of NdpA, a few truncated mutants of UIM were constructed as indicated in Fig. S6A, co-immunoprecipitation analysis data indicated that the two UIMs are dispensable for the polyubiquitination of $\mathrm{NdpA}$ (Fig. S6B), we thus surmised that the UIM motif in NdpA might participate in other currently unknown regulatory functions in hosts.

We next sought to determine the type of ubiquitin chains in $\mathrm{NdpA}$, and we found that $\mathrm{NdpA}$ was effectively polyubiquitinated by Lys 48-linked polyubiquitin (K48 only) chains, but not the non-degradative Lys 63-linked polyubiquitin (K63 only) chains (Fig. 2C). Furthermore, the ubiquitin mutant (K48R) instead of the ubiquitin mutant (K63R) couldn't promote NdpA polyubiquitination (Fig. 2D). Consistently, ubiquitin (K48R) lost the ability to destabilize NdpA protein, while ubiquitin (K63R) promoted degradation of $\mathrm{NdpA}$ as same as the WT ubiquitin (Fig. 2E). These results indicated that NdpA might be degraded through the proteasome pathway since Lys 48-linked polyubiquitination is commonly associated with the proteasome pathway-dependent protein degradation. We then found that MG132, the inhibitor of proteasome, indeed stabilized the NdpA protein (Fig. 2F). Increasing number of evidences revealed that ubiquitin could also target substrates to lysosome for degradation ( $\mathrm{Lu}$ et al., 2014; Pankiv et al., 2007). But our data showed that the lysosome inhibitor chloroquine failed to stabilize NdpA (Fig. 2F). A previous study reported that ubiquitin could mediate ERK1/2 degradation through proteasome-dependent pathway ( $\mathrm{Lu}$ et al., 2002), we thus examined whether NdpA affects ERK1/ 2 polyubiquitination and degradation. Our data showed that overexpression of $\mathrm{NdpA}$ had little effect on the polyubiquitiniatin of ERK2, as well as the endogenous ERK1/2 protein levels (Fig. S7A and S7B). Collectively, K. pneumoniae NdpA undergoes Lys 48-linked polyubiquitination-mediated proteasomal degradation in host cells.

In this study, we revealed that $\mathrm{NdpA}$, a secreted effector protein of K. pneumoniae, specifically inhibits ERK signaling pathway, leading to the suppression of host early inflammatory responses. We found that $\mathrm{NdpA}$ from $K$. pneumoniae abrogates ERK pathway-mediated expression of IL8 in a D motifdependent manner. Sequence alignment results indicate that $\mathrm{NdpA}$ is a highly conserved protein widely exists in gramnegative bacteria, most of them share the same or similar protein sequence of $D$ motif with $K$. pneumoniae $\mathrm{NdpA}$. Therefore, the $D$ motif might provide selectivity for the development of novel pan-anti-gram-negative pathogen therapies.

While in immunocompromised hosts (such as the aged patients) whose innate immunity such as proteasome function is attenuated during aging process (Tonoki et al., 2009), the bacteria might gain the upper hand and cause diseases. Thus our findings help to explain, at least partially, that why K. pneumoniae tends to infect immunocompromised hosts such as the seniors.

\section{FOOTNOTES}

This work was supported by research funding from the National Basic Research Program (973 Program) (Nos. 2014CB744400 and 2012CB518700), the the National Natural Science Foundation of China (Grant Nos. 81371769, 81571954 and 81571536), the Beijing Natural Science Foundation (Grant No. 5162021), and the Youth Innovation Promotion Association CAS (Grant No. Y12A027BB2). We thank Feng Shao (National Institute of Biological Sciences, Beijing, China), Lingqiang Zhang (Beijing Institute of Radiation Medicine) and Youjun Feng (Zhejiang University) for providing our plasmids.

Guanghua Xu, Jing Wang, and Cui Hua Liu declare that they have no conflict of interest. This article does not contain any studies with human or animal subjects performed by the any of the authors.

\section{OPEN ACCESS}

This article is distributed under the terms of the Creative Commons Attribution 4.0 International License (http://creativecommons.org/ licenses/by/4.0/), which permits unrestricted use, distribution, and reproduction in any medium, provided you give appropriate credit to the original author(s) and the source, provide a link to the Creative Commons license, and indicate if changes were made. 
Guanghua $X u^{1,2}$, Jing Wang ${ }^{1}$, Cui Hua Liu ${ }^{1,3 凶}$

${ }^{1}$ CAS Key Laboratory of Pathogenic Microbiology and Immunology, Institute of Microbiology, Chinese Academy of Sciences, Beijing 100101, China

2 School of Life Sciences, Anhui University, Hefei 230601, China

${ }^{3}$ Savaid Medical School, University of Chinese Academy of Sciences, Beijing 101408, China

$\bowtie$ Correspondence: liucuihua@im.ac.cn (C. H. Liu)

\section{REFERENCES}

Frank CG, Reguerio V, Rother M, Moranta D, Maeurer AP, Garmendia J, Meyer TF, Bengoechea JA (2013) Klebsiella pneumoniae targets an EGF receptor-dependent pathway to subvert inflammation. Cell Microbiol 15:1212-1233

Harada A, Sekido N, Akahoshi T, Wada T, Mukaida N, Matsushima K (1994) Essential involvement of interleukin-8 (IL-8) in acute inflammation. J Leukoc Biol 56:559-564

Karaiskos I, Souli M, Galani I, Giamarellou H (2016) Colistin: still a lifesaver for the 21st century? Expert Opin Drug Metab Toxicol 14:1-13

Lawlor MS, Handley SA, Miller VL (2006) Comparison of the host responses to wild-type and cpsB mutant Klebsiella pneumoniae infections. Infect Immun 74:5402-5407

Lu Z, Xu S, Joazeiro C, Cobb MH, Hunter T (2002) The PHD domain of MEKK1 acts as an E3 ubiquitin ligase and mediates ubiquitination and degradation of ERK1/2. Mol Cell 9:945-956
Lu K, Psakhye I, Jentsch S (2014) Autophagic clearance of polyQ proteins mediated by ubiquitin-Atg 8 adaptors of the conserved CUET protein family. Cell 158:549-563

Pankiv S, Clausen TH, Lamark T, Brech A, Bruun JA, Outzen H, Overvatn A, Bjorkoy G, Johansen T (2007) p62/SQSTM1 binds directly to Atg8/LC3 to facilitate degradation of ubiquitinated protein aggregates by autophagy. J Biol Chem 282:24131-24145

Park IH, Jun CH, Wi JW, Park SY, Lee WS, Jung SI, Park CH, Joo YE, Kim HS, Choi SK et al (2015) Prevalence of and risk factors for endogenous endophthalmitis in patients with pyogenic liver abscesses. Korean J Intern Med 30:453-459

Polo S, Confalonieri S, Salcini AE, Di Fiore PP (2003) EH and UIM: endocytosis and more. Sci STKE 213:1-17

Regueiro V, Moranta D, Frank CG, Larrarte E, Margareto J, March C, Garmendia J, Bengoechea JA (2011) Klebsiella pneumoniae subverts the activation of inflammatory responses in a NOD1dependent manner. Cell Microbiol 13:135-153

Tonoki A, Kuranaga E, Tomioka T, Hamazaki J, Murata S, Tanaka K, Miura M (2009) Genetic evidence linking age-dependent attenuation of the $26 \mathrm{~S}$ proteasome with the aging process. Mol Cell Biol 29:1095-1106

Zhou T, Sun L, Humphreys J, Goldsmith EJ (2006) Docking interactions induce exposure of activation loop in the MAP kinase ERK2. Structure 14:1011-1019

Zhu Y, Li H, Long C, Hu L, Xu H, Liu L, Chen S, Wang DC, Shao F (2007) Structural insights into the enzymatic mechanism of the pathogenic MAPK phosphothreonine lyase. Mol Cell 28:899-913

Electronic supplementary material The online version of this article (doi:10.1007/s13238-016-0341-y) contains supplementary material, which is available to authorized users. 